\title{
Estrutura do estrato herbáceo de uma restinga arbustiva aberta na APA de Massambaba, Rio de Janeiro, Brasil ${ }^{1}$
}

\section{Herb layer structure of an open scrub restinga in the Massambaba Environmental Protection Area,} Rio de Janeiro, Brazil

\author{
Daniele Andrade de Carvalho ${ }^{2,3} \&$ Cyl Farney Catarino de Sá ${ }^{2}$
}

\begin{abstract}
Resumo
A APA de Massambaba está inserida no Centro de Diversidade Vegetal de Cabo Frio, que se destaca na costa sul-sudeste por sua elevada riqueza de espécies. As restingas, predominantes nessa região, estão sujeitas a estresses ambientais e têm sofrido histórica pressão antrópica. Este trabalho objetivou levantar florística e estruturalmente o estrato herbáceo de uma comunidade arbustiva aberta na APA de Massambaba. O estrato herbáceo foi amostrado através do método de parcelas, totalizando $200 \mathrm{~m}^{2}$. Os parâmetros de frequência e cobertura das espécies foram calculados, assim como os respectivos valores de importância. Os resultados foram comparados com a formação aberta de Clusia (Macaé, RJ), utilizando-se os índices de similaridade de Sorensen (qualitativo e quantitativo), diversidade de Shannon e equabilidade de Pielou. Foram encontradas 33 espécies pertencentes ao estrato herbáceo, que apresentou estrutura oligárquica e as seguintes dominantes: Panicum trinii, Allagoptera arenaria, Vriesea neoglutinosa, Chamaecrista ramosa, Sebastiania glandulosa, Conepia ovalifolia, Diodella apiculata e Cuphea flava. O estrato herbáceo de Jurubatiba foi similar ao de Massambaba $(\mathrm{Cs}=0,59)$ e este último apresentou maior diversidade $\left(\mathrm{H}^{\prime} \mathrm{C}=2,32\right.$ nats $\left./ \mathrm{m}^{2}\right)$.
\end{abstract}

Palavras-chave: estrato herbáceo, fitossociologia, restinga, Rio de Janeiro.

\begin{abstract}
The Massambaba Environmental Protection Area lies within the Cabo Frio Center of Plant Diversity, which has high species richness when compared to the entire Brazilian south-southeast coast. The restingas (sandy coastal plains) predominate in this region and they suffer from environmental stress plus historic manmade activities. This work aims to survey the floristic composition and structure of the herb layer of an open scrub formation in the Massambaba Environmental Protection Area, and compare it to a physionomically similar formation. The herb layer was sampled by the plot technique, totaling $200 \mathrm{~m}^{2}$. The parameters frequency, cover and Importance Value were calculated for each species. For the comparisons with the Clusia scrub formation (Macaé, RJ), similarity indices (Sorensen's qualitative and quantitative), diversity (Shannon) and evenness (Pielou) were used. A total of 33 species were recorded for the herb layer, showing an oligarchic structure with the following dominants: Panicum trinii, Allagoptera arenaria, Vriesea neoglutinosa, Chamaecrista ramosa, Sebastiania glandulosa, Couepia ovalifolia,Diodella apiculata and Cuphea flava. Jurubatiba's herb layer was very similar to Massambaba's $(\mathrm{Cs}=0.59)$; the latter has higher diversity $\left(\mathrm{H}_{\mathrm{C}}{ }_{\mathrm{C}}=2.32\right.$ nats $\left./ \mathrm{m}^{2}\right)$.
\end{abstract}

Key words: herb layer, phytosociology, sandy coastal plain, Rio de Janeiro.

\section{Introdução}

As restingas podem ser definidas como áreas litorâneas constituídas de depósitos quaternários arenosos de origem marinha e dunas construídas sobre estes depósitos pela ação do vento (Araujo \& Maciel 1998), sendo caracterizadas por apresentar uma variedade de comunidades com fauna e flora características (Lacerda et al. 1993). Segundo Scarano (2002), as comunidades periféricas à Mata Atlântica, nas quais se incluem as restingas, estão sujeitas a adversidades abióticas (altas temperaturas, períodos de seca, vento constante, alta salinidade e escassez de nutrientes), que tornam a sua estrutura e função diferentes de quaisquer outros ambientes.

\footnotetext{
${ }^{\mathrm{I}}$ Parte da monografia de Bacharelado da primeira autora.

${ }^{2}$ Instituto de Pesquisas Jardim Botânico do Rio de Janeiro, R. Pacheco Leão 915, 22460-030, Rio de Janeiro, RJ.

${ }^{3}$ Autor para correspondência: daniele.acarvalho@gmail.com.
} 
Somado às adversidades naturais que as restingas estão submetidas, estes ecossistemas vêm sofrendo uma histórica pressão antrópica, sendo área preferencial de ocupação humana desde 8.000 anos A.P. (Kneip \& Pallestrini 1984). Atualmente, os processos de degradação (especulação imobiliária, retirada de areia, fogo, entre outros) a que estas áreas litorâneas estão condicionadas têm ocasionado a perda de extensivas porções deste habitat em um ritmo acelerado, sendo necessárias medidas efetivas de proteção aplicadas a este ecossistema (Rocha et al. 2007).

Apesar das pressões que as restingas têm sofrido, algumas áreas ainda apresentam alta diversidade e endemismo de espécies, como a região de Cabo Frio - RJ, que é reconhecida pelo WWF/IUCN como um dos 14 centros de diversidade vegetal do Brasil (Davis et al. 1997). O Centro de Diversidade Vegetal de Cabo Frio (CDVCF) está situado no domínio da Mata Atlântica e de acordo com Araujo (1997) possui a maior riqueza de espécies das restingas do Rio de Janeiro (57\% das espécies em 12\% da área total).

A Área de Proteção Ambiental (APA) de Massambaba, inserida no CDVCF, é considerada uma das áreas de restinga de extrema importância ecológica e necessita de proteção mais efetiva e urgente (Rocha et al. 2003). Apesar dessa relevância, os poucos trabalhos referentes à estrutura das comunidades vegetais que ali ocorrem, focaram formações florestais (Sá 1996, 2002; Sá \& Araujo 2009; Fonseca-Kruel et al. 2009), salvo um estudo na comunidade arbustiva de Palmae (Almeida \& Araújo 1997), desenvolvido na parte ocidental da APA (Saquarema), havendo uma lacuna para as demais comunidades, especialmente as arbustivas abertas que ocorrem nos municípios de Araruama e Arraial do Cabo.

As restingas arbustivas abertas caracterizamse pela organização em ilhas de vegetação de diferentes tamanhos circundadas por areia nua (Scarano et al. 2004). Nessas áreas de areia nua ocorre uma vegetação esparsa de espécies herbáceassubarbustivas, sendo geralmente diferentes das espécies que compõem o estrato herbáceo das moitas (Araujo et al. 2009). Citadini-Zanete \& Baptista (1989) citaram as espécies herbáceas como indicadoras das condições ambientais, devido ao seu pequeno porte e sistema radicular superficial, o que as tornam particularmente sensíveis às alterações do microclima e do solo. Em florestas tropicais, foi apontado que até pequenos distúrbios na vegetação podem alterar consideravelmente a diversidade de espécies herbáceas (Yadav \& Gupta 2007).

Nas restingas fluminenses já foram realizados alguns estudos taxonômicos (Freitas 1992; Sarahyba 1993; Fraga et al. 2005) e ecológicos (Rocha-Pessôa et al. 2008) enfocando famílias botânicas herbáceas, assim como outros identificaram diversos tipos de formações (Silva \& Somner 1984; Henriques et al. 1986; Almeida \& Araujo 1997; Menezes \& Araujo 1999; Assumpção \& Nascimento 2000), nas quais as espécies herbáceas e subarbustivas estão bem representadas. Entretanto, poucos trabalhos têm focado a composição e estrutura do estrato herbáceo (Sá 1996; Pereira et al. 2004; Cordeiro 2005), sendo estes conhecimentos fundamentais para o melhor entendimento das diferentes comunidades de restinga, permitindo elaborar estratégias de conservação que levem em consideração as particularidades de cada área.

O objetivo deste trabalho foi descrever florística e estruturalmente o estrato herbáceo de um trecho da comunidade arbustiva aberta não inundável (fácies alta) da APA de Massambaba, e compará-lo com uma formação fisionomicamente semelhante, a comunidade aberta de Clusia, no Parque Nacional da Restinga de Jurubatiba, através do estudo de Pereira et al. (2004).

\section{Material e Métodos}

Área de estudo

O trabalho foi realizado num trecho de restinga da Área de Proteção Ambiental (APA) de Massambaba, Arraial do Cabo, RJ, entre as coordenadas $22^{\circ} 55^{\prime}-22^{\circ} 56^{\prime} \mathrm{S}$ e $42^{\circ} 14^{\prime}-42^{\circ} 12^{\prime} \mathrm{W}$ (Fig. 1). A APA está inserida no Centro de Diversidade Vegetal de Cabo Frio, com área de $1.562 \mathrm{~km}^{2}$ (Bohrer et al. 2009) e apresenta alta diversidade florística, endemismos e espécies ameaçadas de extinção (Araujo 1997). Possui reduzida precipitação $(823 \mathrm{~mm}$ anuais), temperatura média anual entre $18^{\circ}$ e $23^{\circ} \mathrm{C}$, sendo o calor distribuído o ano todo, e o clima local foi classificado como uma variação do Clima SemiÁrido Quente (BSh) (Barbiere 1984). De acordo com Bohrer et al. (2009), a área do CDVCF ocupada por restinga arbustiva corresponde a 1.451,4 ha e ocorre em geral sobre Neossolos Quartzarênicos.

A Restinga da Massambaba possui $48 \mathrm{~km}$ de extensão pela linha da costa e situa-se nos municípios de Saquarema, Araruama e Arraial do Cabo. Apresenta dois cordões arenosos separados 


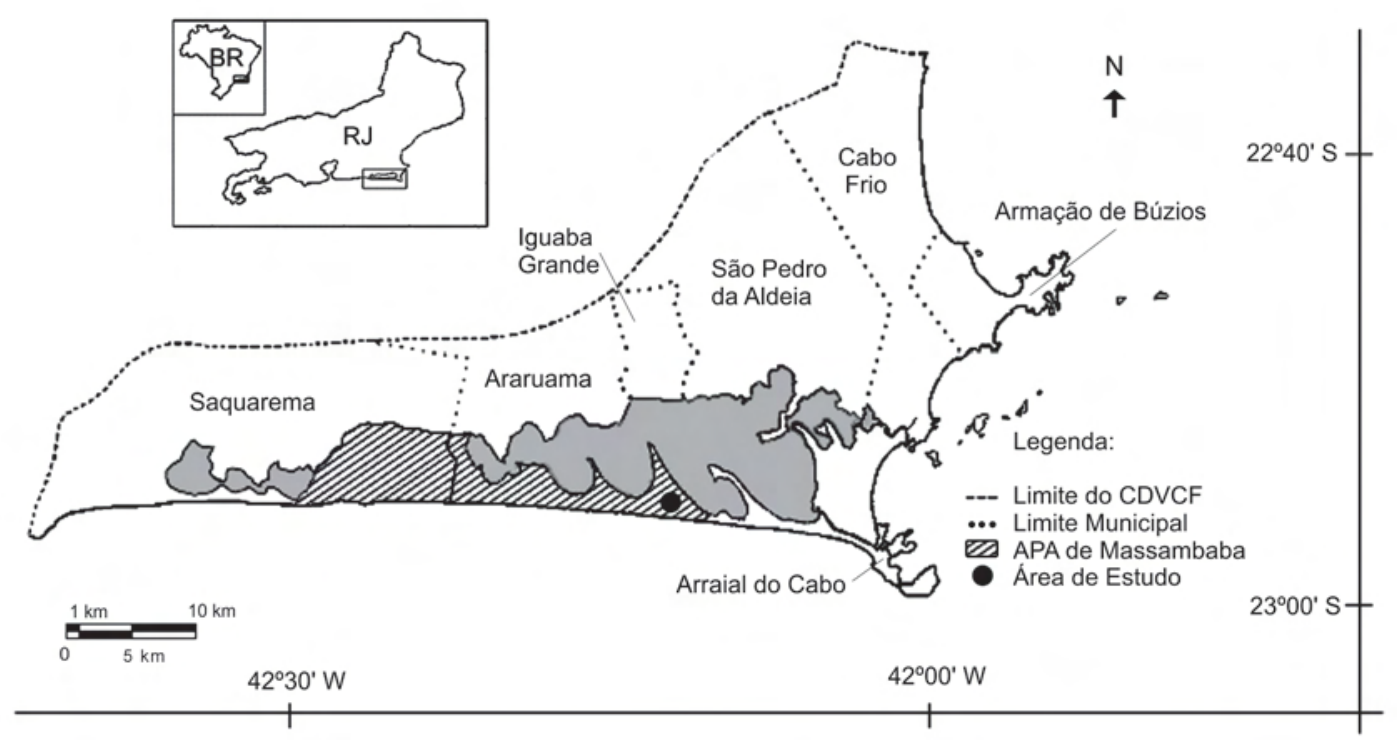

Figura 1 - Localização da área de estudo no Centro de Diversidade Vegetal de Cabo Frio, RJ (Fonte: adaptado de Soares-Gomes \& Fernandes 2005; Araujo et al. 2009).

Figure 1 - Location of the study area within Cabo Frio Center of Plant Diversity, RJ (Source: adapted from Soares-Gomes \& Fernandes 2005; Araujo et al. 2009).

por uma faixa de relevo mais baixo, com uma interrupção do cordão mais antigo na extremidade oriental da restinga, onde se localiza a área de estudo, sendo o cordão frontal a única barreira entre a lagoa de Araruama e o oceano (Muehe 1994).

A APA foi criada por decreto estadual em 1986 e ainda conserva remanescentes dos ecossistemas de restinga, manguezal, laguna e brejo pouco alterados pelo homem, além de importantes sambaquis, que registram a presença de grupos pré-históricos na área (SEMADS/RJ 2001). Entretanto, dentre as restingas da costa do estado, a região da Massambaba é a que se encontra sob maior pressão antrópica, sendo as principais causas de destruição local, a expansão imobiliária, o asfaltamento das vias de acesso e o uso indiscriminado do lençol freático (Rocha et al.2009).

A comunidade vegetal em estudo foi classificada por Araujo et al. (2009) como Formação Arbustiva Aberta não Inundável - fácies alta, e ocorre na extremidade oriental da APA, onde a maior parte do cordão interno é coberta por um extenso campo de dunas fixas, com relevo muito variado. Esta formação é caracterizada por moitas de diversos tamanhos, com até $5 \mathrm{~m}$ de altura e cobertura relativamente esparsa de plantas herbáceas ou subarbustivas entre as moitas. Entre as principais espécies lenhosas que compõem as moitas, destacam-se: Couepia ovalifolia (Schott) Benth., Maytenus obtusifolia Mart., Myrsine parvifolia A. DC e Erythroxylum ovalifolium Peyr.

\section{Análise florística e estrutural}

Para a análise estrutural da vegetação do estrato herbáceo, foi utilizado o método de parcelas (Brower et al. 1998). Foram inventariados dois pontos de amostragem, separados por aproximadamente $400 \mathrm{~m}$. Em cada ponto, foram lançadas 20 linhas de $50 \mathrm{~m}$, com distância de $20 \mathrm{~m}$ entre si, orientadas perpendicularmente ao mar. A cada $10 \mathrm{~m}$ dessa linha, foi sorteada uma parcela de $1 \mathrm{~m}^{2}$ para alocação à esquerda ou à direita da linha, sendo amostradas cinco parcelas por linha, 100 parcelas por ponto amostral e totalizando $200 \mathrm{~m}^{2}$ inventariados. As parcelas foram alocadas tanto nas moitas como na área entre-moitas.

Foram estimados, por parcela, os percentuais de cobertura vegetal para cada espécie do estrato herbáceo, da cobertura vegetal total (sem distinção entre cobertura vegetal de herbáceas, subarbustivas e arbustivas), da área coberta por detritos e da área nua. Como detritos foram considerados elementos vegetais destacados dos corpos dos indivíduos como galhos, folhas, frutos, sementes e flores; conchas e outros restos animais que ocorriam na parcela. 
Na área de moitas, uma vez que o solo era praticamente todo coberto por folhas secas e outros detritos vegetais, o percentual de detritos correspondeu a $100 \%$, ocorrendo sobreposição com o percentual de cobertura vegetal total. Os percentuais foram estimados visualmente com auxílio de um quadrado de $0,5 \times 0,5 \mathrm{~m}$ dividido com fios de nylon em 25 quadrados, cada um correspondendo a $1 \%$ da área.

Como pertencente ao estrato herbáceo, foram considerados todos os subarbustos e ervas, de acordo com Pereira et al. (2004), sendo os hábitos das espécies determinados a partir de observações de campo e posterior consulta ao especialista e/ou literatura especializada. Também foram incluídos na amostragem os indivíduos menores que $50 \mathrm{~cm}$ de altura das espécies tipicamente arbustivas Couepia ovalifolia, Gaylussacia brasiliensis e Stigmaphyllon paralias, pois estes ocorrem frequentemente nos espaços entre as moitas, apresentando pequeno porte e assemelhando-se a subarbustos. As espécies Epidendrum denticulatum e E. orchidiflorum foram consideradas uma só morfo-espécie devido à incapacidade de diferenciá-las por material vegetativo.

Foram calculados os parâmetros fitossociológicos de frequência (absoluta e relativa), cobertura (absoluta e relativa), e Valor de Importância (VI) para cada espécie encontrada (Brower et al. 1998). A densidade não foi calculada devido à dificuldade em diferenciar indivíduos distintos entre os rametes das espécies rizomatozas ou estoloníferas, muito frequentes na amostra. Este procedimento tem sido adotado em outros trabalhos estruturais de restinga (Menezes \& Araujo 1999; Assumpção \& Nascimento 2000; Cordeiro 2005) sendo o Valor de Importância baseado nos valores relativos de freqüência e dominância (cobertura vegetal). De acordo com
Muller-Dombois \& Ellenberg (1974), a escolha dos parâmetros fitossociológicos que serão utilizados no cálculo do Valor de Importância depende dos valores que o pesquisador julga serem mais importantes para compreender a estrutura da comunidade ou do grupo de espécies em estudo.

Com base no Valor de Importância, as espécies foram classificadas em dominantes (VI $>10)$, intermediárias $(10>\mathrm{VI}>1)$ e raras $(\mathrm{VI}<1)$, como proposto por Pereira et al. (2004). Deve-se ressaltar que o VI calculado por essas autoras foi a partir dos parâmetros relativos de frequiência, cobertura e densidade, com o total somando 300 , enquanto que o presente trabalho utilizou apenas os dois primeiros parâmetros, sendo o somatório do VI igual a 200. Mas, levando-se em consideração o acima exposto, que os parâmetros de frequiência e cobertura relativas são suficientes para compreender a estrutura do estrato em estudo, considerou-se como apropriada a utilização dessa mesma classificação das espécies, apesar de ter restringido algumas comparações diretas com o trabalho de Pereira et al. (2004).

A diversidade foi estimada através do índice de Shannon (H') e da equabilidade de Pielou (J), nos quais os valores de frequência e cobertura foram utilizados como medidas de abundância das espécies (Magurran 1988).

Devidoàs semelhanças metodológicas (definição do estrato herbáceo como ervas + subarbustos e utilização de parcelas de $1 \mathrm{~m}^{2}$ ), esse estudo foi comparado com o de Pereira et al. (2004), cujo foco foi analisar o estrato herbáceo de uma comunidade de restinga em moitas no Parque Nacional (PARNA) da Restinga de Jurubatiba (Tab. 1). Foram calculados os índices de similaridade de Sorensen (Cs) e Sorensen quantitativo $(\mathrm{CN})$, sendo o número de indivíduos substituído pelo

Tabela 1 - Comparação entre a comunidade arbustiva aberta não inundável (fácies alta), na APA de Massambaba, RJ e a formação arbustiva aberta de Clusia, no Parque Nacional da Restinga de Jurubatiba, RJ.

Table 1 - Comparisons between the non-flooded open scrub formation in the APA of Massambaba, RJ and the Clusia open scrub in the Jurubatiba National Park, RJ.

\begin{tabular}{lll}
\hline & Este trabalho & Pereira et al. (2004) \\
\hline Localidade & APA de Massambaba & PARNA da Restinga de Jurubatiba \\
Comunidade estudada & Arbustiva aberta nãoinundável (fácies alta) & Arbustiva aberta de Clusia \\
Critério de inclusão & ervas + subarbustos & ervas + subarbustos \\
Método amostragem & parcelas $1 \mathrm{~m}^{2}$ & parcelas $1 \mathrm{~m}^{2}$ \\
Total amostrado & $200 \mathrm{~m}^{2}$ & $600 \mathrm{~m}^{2}$ \\
Número de espécies & 33 & 39 \\
Família mais rica em espécies & Leguminosae & Rubiaceae \\
Diversidade - H' (cobertura) & 2,32 nats $/ \mathrm{m}^{2}$ & 1,89 nats $/ \mathrm{m}^{2}$ \\
Equabilidade - J' (cobertura) & 0,67 & 0,52 \\
\hline
\end{tabular}


valor de cobertura das espécies (Magurran 1988). Para comparar os índices de Shannon com base na cobertura $\left(\mathrm{H}^{\prime} \mathrm{C}\right)$, foi utilizado o método de Hutcheson para o cálculo do teste t, como recomenda Zar (1999).

O material botânico testemunho foi coletado, herborizado e depositado no Herbário do Instituto de Pesquisas Jardim Botânico do Rio de Janeiro (RB). A identificação taxonômica foi realizada através de consulta à literatura especializada, comparação com material de herbário e, sempre que possível, as espécies foram confirmadas pelos especialistas de cada família botânica. Para a classificação taxonômica das famílias de angiospermas, adotou-se o
Angiosperm Phylogeny Group II (APG II 2003), exceto para Leguminosae, que seguiu Lewis et al. (2005). As pteridófitas foram classificadas de acordo com Smith et al. (2006).

\section{Resultados}

Foram encontradas 33 espécies no estrato herbáceo da comunidade estudada, sendo distribuídas em 19 famílias e 28 gêneros (Tab. 2). A família com maior número de espécies foi Leguminosae (4), seguida por Cactaceae, Rubiaceae, Orchidaceae (3), Araceae, Bromeliaceae, Cyperaceae, Poaceae e Verbenaceae (2).

Tabela 2 - Lista das espécies do estrato herbáceo da comunidade arbustiva aberta da APA de Massambaba, RJ, organizadas em ordem alfabética de famílias. Sigla do coletor: DAC = Daniele Andrade de Carvalho.

Table 2 - Species list from the herb layer of the non-flooded open scrub formation in the APA of Massambaba, RJ, alphabetic ordered by families. Abbreviation for collector: DAC $=$ Daniele Andrade de Carvalho.

\begin{tabular}{|c|c|c|}
\hline Família & Espécie & No. coletor \\
\hline Araceae & Anthurium maricense Nadruz \& Mayo & DAC 64 \\
\hline Araceae & Philodendron corcovadense Kunth & DAC 124 \\
\hline Arecaceae & Allagoptera arenaria (Gomes) Kuntze & DAC 125 \\
\hline Asteraceae & Trichogoniopsis podocarpa (DC.) R.M. King \& H. Rob. & DAC 83 \\
\hline Bromeliaceae & Neoregelia cruenta (Graham) L. B. Sm. & $\mathrm{s} / \mathrm{n}^{\circ}$ \\
\hline Bromeliaceae & Vriesea neoglutinosa $\mathrm{Mez}$ & DAC 65 \\
\hline Cactaceae & Cereus fernambucensis Lem. & DAC 69 \\
\hline Cactaceae & Melocactus violaceus Pfeiff. & DAC 123 \\
\hline Cactaceae & Pilosocereus arrabidae (Lem.) Byles \& G. D. Rowley & DAC 77 \\
\hline Chrysobalanaceae & Couepia ovalifolia (Schott) Benth. & DAC 52 \\
\hline Convolvulaceae & Evolvulus genistoides Ooststr. & DAC 74 \\
\hline Cyperaceae & Abildgaardia baeothryon A. St.-Hil. & DAC 55 \\
\hline Cyperaceae & Lagenocarpus rigidus Nees & DAC 68 \\
\hline Ericaceae & Gaylussacia brasiliensis (Spreng.) Meisn. & DAC 72 \\
\hline Euphorbiaceae & Sebastiania glandulosa (Mart.) Pax & DAC 51 \\
\hline Leguminosae & Chamaecristaflexuosa (L.) Greene & DAC 54 \\
\hline Leguminosae & Chamaecrista ramosa (Vogel) H. S. Irwin \& R. C. Barneby & DAC 63 \\
\hline Leguminosae & Stylosanthes guianensis (Aubl.) Sw. & DAC 62 \\
\hline Leguminosae & Stylosanthes viscosa (L.) Sw. & DAC 76 \\
\hline Lythraceae & Cuphea flava Spreng. & DAC 53 \\
\hline Malpighiaceae & Stigmaphyllon paralias A. Juss. & DAC 93 \\
\hline Mollugaceae & Mollugo verticillata $\mathrm{L}$. & DAC 70 \\
\hline Orchidaceae & Epidendrum denticulatum Barb. Rodr. & DAC 56 \\
\hline Orchidaceae & Epidendrum orchidiflorum (Salzm.) Salzm. ex Lindl & DAC 82 \\
\hline Orchidaceae & Vanilla cf. chamissonis Klotzsch & $\mathrm{s} / \mathrm{n}^{\circ}$ \\
\hline Poaceae & Indeterminada & $\mathrm{s} / \mathrm{n}^{\circ}$ \\
\hline Poaceae & Panicum trinii Kunth & DAC 81 \\
\hline Polypodiaceae & Serpocaulon triseriale (Sw.) A.R. Sm. & DAC 86 \\
\hline Rubiaceae & Diodella apiculata (Willd. ex Roem. \& Schult.) Delprete & DAC 59 \\
\hline Rubiaceae & Diodella radula (Willd. ex Roem. et Schult.) Delprete & DAC 84 \\
\hline Rubiaceae & Mitracarpus lhotzkyanus Cham. & DAC 50 \\
\hline Verbenaceae & Lantana fucata Lindl. & DAC 85 \\
\hline Verbenaceae & Stachytarpheta restingensis Moldenke & DAC 71 \\
\hline
\end{tabular}


No levantamento de dados da estrutura da comunidade, a cobertura vegetal total correspondeu a 71,6\% da área $\left(143,27 \mathrm{~m}^{2}\right)$, sendo a cobertura de detritos de $56,3 \%\left(28,14 \mathrm{~m}^{2}\right)$ e a de areia nua de $12,3 \%\left(24,75 \mathrm{~m}^{2}\right)$, considerando a sobreposição dos percentuais de detritos e vegetação.

A análise estrutural da vegetação incluiu 32 espécies, ao considerar Epidendrum denticulatum e E. orchidiflorum como uma única morfo-espécie
(Tab. 3). As espécies que apresentaram maiores valores de importância foram Panicum trinii, Allagoptera arenaria, Vriesea neoglutinosa, Chamaecrista ramosa, Sebastiania glandulosa, Couepia ovalifolia, Diodella apiculata, e Cuphea flava. Essas oito espécies foram consideradas dominantes (75\% do VI), 13 espécies foram definidas como intermediárias (22\% do VI) e as raras (11 spp.) somaram $2 \%$ do VI.

Tabela 3 - Parâmetros fitossociológicos das espécies do estrado herbáceo da comunidade arbustiva aberta da APA de Massamababa, RJ, organizadas em ordem decrescente de valor de importância. NP: número de parcelas com ocorrência da espécie, CT: cobertura total da espécie $\left(\mathrm{m}^{2}\right)$, FA: frequência absoluta, FR: frequência relativa (\%), CA: cobertura absoluta $\left(\mathrm{m}^{2} / \mathrm{ha}\right), \mathrm{CR}$ : cobertura relativa (\%), VI: valor de importância.

Table 3 - Phytosociological parameters of the species from the herb layer of the non-flooded open scrub formation in the APA of Massambaba, RJ, ordered by highest importance values. NP: number of plots where the species occur, CT: total cover of the species $\left(\mathrm{m}^{2}\right)$, FA: absolute frequency, FR: relative frequency (\%), CA: absolute cover ( $\left.\mathrm{m}^{2} / \mathrm{ha}\right)$, CR: relative cover $(\%)$, VI: importance value.

\begin{tabular}{|c|c|c|c|c|c|c|c|}
\hline Espécie & NP & CT & FA & FR & $\mathbf{C A}$ & $\mathbf{C R}$ & VI \\
\hline Panicum trinii & 100 & 46,92 & 0,500 & 11,82 & 2346,0 & 32,57 & 44,39 \\
\hline Allagoptera arenaria & 103 & 22,02 & 0,515 & 12,17 & 1101,0 & 15,28 & 27,46 \\
\hline Vriesea neoglutionosa & 44 & 17,54 & 0,220 & 5,20 & 877,0 & 12,17 & 17,37 \\
\hline Chamaecrista ramosa & 73 & 10,6 & 0,365 & 8,63 & 530,0 & 7,36 & 15,99 \\
\hline Sebastiania glandulosa & 77 & 6,71 & 0,385 & 9,10 & 335,5 & 4,66 & 13,76 \\
\hline Couepia ovalifolia & 45 & 8,01 & 0,225 & 5,32 & 400,5 & 5,56 & 10,88 \\
\hline Diodella apiculata & 56 & 5,65 & 0,280 & 6,62 & 282,5 & 3,92 & 10,54 \\
\hline Cuphea flava & 47 & 6,97 & 0,235 & 5,56 & 348,5 & 4,84 & 10,39 \\
\hline Evolvulus genistoides & 49 & 2,97 & 0,245 & 5,79 & 148,5 & 2,06 & 7,85 \\
\hline Stigmaphyllon paralias & 44 & 1,23 & 0,220 & 5,20 & 61,5 & 0,85 & 6,05 \\
\hline Cereus fernambucensis & 34 & 2,12 & 0,170 & 4,02 & 106,0 & 1,47 & 5,49 \\
\hline Anthurium maricense & 27 & 2,27 & 0,135 & 3,19 & 113,5 & 1,58 & 4,77 \\
\hline Mollugo verticillata & 22 & 0,67 & 0,110 & 2,60 & 33,5 & 0,47 & 3,07 \\
\hline Chamaecrista flexuosa & 21 & 0,82 & 0,105 & 2,48 & 41,0 & 0,57 & 3,05 \\
\hline Epidendrum spp. & 11 & 2,44 & 0,055 & 1,30 & 122,0 & 1,69 & 2,99 \\
\hline Pilosocereus arrabidae & 16 & 1,42 & 0,080 & 1,89 & 71,0 & 0,99 & 2,88 \\
\hline Melocactus violaceus & 19 & 0,41 & 0,095 & 2,25 & 20,5 & 0,28 & 2,53 \\
\hline Stachytarpheta restingensis & 10 & 0,5 & 0,050 & 1,18 & 25,0 & 0,35 & 1,53 \\
\hline Vanilla cf.chamisonis & 6 & 1,04 & 0,030 & 0,71 & 52,0 & 0,72 & 1,43 \\
\hline Stylosanthes guianensis & 9 & 0,3 & 0,045 & 1,06 & 15,0 & 0,21 & 1,27 \\
\hline Mitracarpus lhotzkyanus & 9 & 0,22 & 0,045 & 1,06 & 11,0 & 0,15 & 1,22 \\
\hline Lagenocarpus rigidus & 3 & 0,82 & 0,015 & 0,35 & 41,0 & 0,57 & 0,92 \\
\hline Gaylussacia brasiliensis & 5 & 0,08 & 0,025 & 0,59 & 4,0 & 0,06 & 0,65 \\
\hline Poaceae indeterminada & 1 & 0,66 & 0,005 & 0,12 & 33,0 & 0,46 & 0,58 \\
\hline Neorogelia cruenta & 1 & 0,59 & 0,005 & 0,12 & 29,5 & 0,41 & 0,53 \\
\hline Diodella radula & 3 & 0,24 & 0,015 & 0,35 & 12,0 & 0,17 & 0,52 \\
\hline Serpocaulon triseriale & 2 & 0,29 & 0,010 & 0,24 & 14,5 & 0,20 & 0,44 \\
\hline Philodendron corcovadensis & 2 & 0,22 & 0,010 & 0,24 & 11,0 & 0,15 & 0,39 \\
\hline Trichogoniopis podocarpa & 2 & 0,15 & 0,010 & 0,24 & 7,5 & 0,10 & 0,34 \\
\hline Lantana fucata & 2 & 0,1 & 0,010 & 0,24 & 5,0 & 0,07 & 0,31 \\
\hline Abildgaardia baeothryon & 2 & 0,09 & 0,010 & 0,24 & 4,5 & 0,06 & 0,30 \\
\hline Stylosanthes viscosa & 1 & 0,01 & 0,005 & 0,12 & 0,5 & 0,01 & 0,13 \\
\hline Total & 846 & 144,08 & 4,23 & 100 & 7204 & 100 & 200 \\
\hline
\end{tabular}


Os valores dos índices de Shannon e de equabilidade de Pielou baseados na cobertura das espécies foram, respectivame nte, $\mathrm{H}_{\mathrm{C}}{ }_{\mathrm{C}}=2,32 \mathrm{nats} / \mathrm{m}^{2}$ e $\mathbf{J}^{\prime}{ }_{\mathrm{C}}=0,67$, e os valores baseados na frequência foram $\mathrm{H}_{\mathrm{F}}=2,90 \mathrm{e} \mathrm{J}_{\mathrm{F}}=0,84$. Na comparação deste trabalho como de Pereira et al. (2004), foramencontradas 21 espécies em comum $(65,6 \%$ das espécies aqui amostradas), sendo obtidos os valores dos índices de similaridade de Sorensen $(\mathrm{Cs}=0,59)$ e de Sorensen quantitativo $(\mathrm{CN}=0,64)$. Os valores do índice de Shannon foram significativamente diferentes $(\mathrm{t}=14,38 ; \mathrm{p}<0,001)$, confirmando que a diversidade de espécies de Massambaba é superior à diversidade de Jurubatiba, para o estrato herbáceo das comunidades analisadas. As demais comparações entre ambos os estudos estão apresentadas na Tabela 1.

\section{Discussão}

\section{Estrutura do estrato herbáceo}

O estrato herbáceo da comunidade estudada é formado majoritariamente por ervas e subarbustos, com alguns arbustos de pequeno porte também contribuindo para a sua estrutura. Adicionalmente às espécies aqui levantadas, ocorrem poucos indivíduos de Tillandsia stricta Sol. fixados ao solo mas que não foram considerados na amostragem deste estudo, pois a espécie apresenta hábito predominantemente epifítico. A cobertura vegetal estimada $(71,6 \%)$ não se apresenta de forma contínua, havendo áreas de moitas dominadas por espécies arbustivas, onde a cobertura vegetal chega a $100 \%$, sendo intercaladas por áreas de areia nua e vegetação esparsa, com predomínio de espécies herbáceas e subarbustivas. Como não foram distinguidos os percentuais correspondentes ao estrato herbáceo e ao estrato arbustivo para essa estimativa da cobertura vegetal total, não é possível apontar a contribuição da cobertura de cada estrato, separadamente, para a fisionomia da comunidade estudada.

Devido à maior disponibilidade de nutrientes, de água no solo, e às condições microclimáticas favoráveis que as moitas proporcionam (Zaluar \& Scarano 2000), algumas espécies são restritas à ocorrência em seu interior (Anthurium maricense, Philodendron corcovadense, Epidendrum denticulatum, E. orchidiflorum, Vanilla cf. chamissonis, Serpocaulon triseriale), e outras espécies que suportam as condições mais extremas nas áreas entre-moitas são encontradas predominantemente na vegetação esparsa, podendo ocorrer raramente nas moitas mais abertas (Diodella apiculata, Stachytarpheta restingensis, Melocactus violaceus, Evolvulus genistoides, Gaylussacia brasiliensis, Sebastiania glandulosa, Chamaecrista ramosa, Chamaecrista flexuosa, Cuphea flava, Mollugo verticillata). Apesar de certas espécies serem comuns às duas condições, é perceptível o melhor estabelecimento em um dos hábitats. Isso acontece tanto com espécies típicas da área aberta, como Allagoptera arenaria, que também ocorre em moitas em início de formação, sugerindo que seus indivíduos se desenvolveram sob a areia nua e possibilitaram o estabelecimento de espécies arbustivas ao entorno, ou com espécies características de moitas (Vriesea neoglutinosa, Pilosocereus arrabidae, Cereus fernambucensis), que ao ocorrer sob a vegetação esparsa ou em adensamentos de A. arenaria, podem estar indicando início de formação de moitas nestes locais.

A compreensão a respeito da formação e sucessão em moitas de restinga no $\mathrm{RJ}$ tem sido alvo de pesquisas (Zaluar \& Scarano 2000) e Scarano et al. (2004) sugeriram um modelo de estabilidade dinâmica no sistema de moitas da restinga aberta de Clusia, no PARNA da Restinga de Jurubatiba. Estes autores indicaram que "as propriedades da comunidade vegetal devem manter-se dinamicamente estáveis dentro de ampla escala temporal, uma vez que não há evidências de avanço linear no projeto sucessional". Apesar das diferenças funcionais entre as espécies que compõem ambas as formações (Araujo et al. 2009), é possível que processo semelhante esteja ocorrendo na comunidade arbustiva aberta da APA de Massambaba, sendo fundamental o estudo do estrato herbáceo no entendimento do crescimento e retração das moitas de vegetação. Como apontado por Citadini-Zanette \& Baptista (1989), as espécies herbáceas são particularmente sensíveis a diferenças ambientais, às quais os vegetais de maior porte não manifestam reação. Assim, estudos que relacionem os efeitos microclimáticos, criados pela expansão ou retração de moitas, sob as espécies constituintes do estrato herbáceo podem ser fontes de informações relevantes sobre a dinâmica de moitas de restinga.

A partir da análise estrutural da vegetação, o estrato estudado apresentou dominância de poucas espécies, uma vez que somente oito espécies (25\% do total), somaram $75 \%$ do Valor de Importância (Tab. 3), sugerindo uma estrutura oligárquica para a comunidade. Tal padrão tem sido encontrado para outras restingas do Rio de Janeiro, tanto quando 
considerado o estrato herbáceo (Pereira et al. 2004; Cordeiro 2005) como o lenhoso (Assumpção \& Nascimento 2000; Pereira et al. 2001; Araujo et al. 2004; Montezuma \& Araujo 2007).

Panicum trinii foi a espécie com o maior VI (44,39\%), principalmente devido à elevada cobertura relativa, apresentando o dobro do valor deste parâmetro em relação à Allagoptera arenaria, a segunda espécie de maior VI. Essa espécie forma touceiras no espaço entre moitas que, de acordo com Araujo et al. (2009) tende a morrer do centro para fora, muitas vezes deixando formado um círculo perfeito com a parte viva da planta. Alves et al. (2007) indicaram que $P$. trinii apresenta distribuição geográfica disjunta restinga - campos rupestres, sendo encontrada em Minas Gerais e Bahia, além das restingas. Segundo Cerqueira (2000) esse quadro de distribuição disjunta, comum às espécies que ocorrem no Rio de Janeiro, sugere que a vegetação de restinga não evoluiu como uma comunidade altamente conectada, havendo entradas de espécies individualizadas neste ecossistema ao longo de sucessivos períodos de transgressão e regressão marinha. No Estado do Rio de Janeiro, P. trinii apresenta distribuição restrita, ocorrendo na Massambaba e na restinga de Jacarepaguá (Araujo 2000).

Allagoptera arenaria, que apresentou o segundo maior VI neste trabalho $(27,46 \%)$, é uma espécie típica das formações abertas de restinga ao longo do litoral do estado (Almeida \& Araujo 1997; Menezes \& Araujo 1999; Assumpção \& Nascimento 2000; Pereira et al. 2004; Montezuma \& Araujo 2007), possuindo elevado sucesso regenerativo devido à sua forma de vida geófita (Almeida \& Araujo 1997). Scarano et al. (2004) a indicaram como espécie chave no funcionamento e estrutura da restinga aberta de Clusia, cujo ingresso de maior número de espécies vegetais e formação de moitas são condicionados pela ocorrência dessa palmeira. Apesar das diferenças funcionais entre as duas comunidades, A. arenaria parece atuar também como facilitadora na formação arbustiva aberta de Massambaba, sendo comumente observado o recrutamento de plântulas de outras espécies próximas aos indivíduos de $A$. arenaria provavelmente devido às modificações no ambiente proporcionadas por esta espécie (Menezes \& Araujo 2004). Vale apontar que esse padrão não foi observado em formações em regeneração próximas à área estudada, tanto em floresta de restinga (Sá 1996, 2002), como em restinga arbustiva aberta (Cirne et al. 2003), mas em ambas as áreas houve forte interferência humana (perturbação por tratores na primeira e queima da cobertura vegetal na segunda), em contraste com a comunidade aqui estudada, na qual não têm ocorrido perturbações antrópicas significativas. De qualquer modo, são necessários estudos locais de dinâmica de moitas para confirmar a atuação de Allagoptera arenaria como espécie focal na formação arbustiva aberta da APA de Massambaba.

As bromélias também têm sido apontadas como espécies chave das comunidades de restinga (Zaluar \& Scarano 2000), pois os indivíduos terrestres oferecem um mecanismo para enriquecimento do solo, pelo aumento significativo do conteúdo de matéria orgânica sob sua cobertura (Hay \& Lacerda 1984). Vriesea neoglutinosa, espécie com terceiro maior VI (17,37\%), ocorre em adensamentos robustos, a partir de crescimento clonal, o que explica sua elevada cobertura vegetal. Essa distribuição agregada de $V$. neoglutinosa e de outras bromélias da restinga de Jurubatiba foi relacionada com a presença de moitas por Freitas et al. (2000). Neoregelia cruenta também foi relatada como espécie focal na mesma restinga (Scarano et al. 2004), mas ocorreu com apenas um indivíduo neste estudo. Dentre as Bromeliaceae da formação fechada do pós-praia da APA de Massambaba, essa espécie apresentou a maior abundância da família (Rocha-Pessôa et al. 2008), sugerindo que, apesar de $N$. cruenta ter ocorrido na comunidade arbustiva aberta, esta formação não possui as condições necessárias para seu estabelecimento.

Leguminosae é considerada a família com maior riqueza de espécies das restingas do Rio de Janeiro (Araujo 2000) e destaca-se em levantamentos da flora da Mata Atlântica, principalmente quando considerado o componente arbóreo (Ribeiro \& Lima 2009). A maior riqueza de espécies dessa família no presente estudo ressalta a representatividade de Leguminosae também para o estrato herbáceo das restingas. Chamaecrista ramosa apresentou elevada frequiência, sendo comum na área entre moitas, ocorrendo sobre a areia nua, assim como Chamaecrista flexuosa, espécie comum a outras restingas arbustivas abertas do Rio de Janeiro (Almeida \& Araujo 1997; Menezes \& Araujo 1999; Pereira et al. 2004). Três das quatro espécies aqui levantadas (Chamaecrista flexuosa, Stylosanthes guianensis e Stylosanthes viscosa) foram consideradas por Araujo (2000) como exóticas e/ou ruderais, sendo encontradas, nas restingas, em 
ambientes altamente alterados pelo homem. O eficiente estabelecimento destas espécies e da família como um todo nas planícies arenosas costeiras pode estar relacionado com a capacidade das mesmas ocuparem solos pobres em nutrientes (Campello 1998), característicos das formações arbustivas abertas.

\section{Similaridade com a formação aberta de Clusia}

A similaridade entre a comunidade aqui analisada e aquela estudada por Pereira et al. (2004), no PARNA da Restinga de Jurubatiba, é considerada alta $(\mathrm{Cs}=59 \%)$, já que de acordo com Felfili et al. (2001), índices de Sorensen acima de $50 \%$ indicam elevada similaridade entre duas áreas. Não só houve um elevado número de espécies em comum (21 espécies), como também a cobertura total dessas foi bastante similar $(\mathrm{CN}=64 \%)$.

Araujo et al. (2009) salientaram a cautela na comparação de comunidades de restinga fisionomicamente equivalentes, exemplificando as formações arbustiva aberta não inundável (fácies alta), e a arbustiva aberta de Clusia. Os autores ressaltaram que as moitas da formação encontrada em Massambaba não são estruturadas tendo como dominante a espécie Clusia hilariana, que atua como facilitadora para o estabelecimento de outras espécies em Jurubatiba (Scarano et al. 2004), indicando que a semelhança fisionômica entre as duas formações não significa equivalência das características funcionais das espécies. Portanto, tal premissa orientou as comparações realizadas entre essas duas comunidades, assim como as demais comparações realizadas neste trabalho.

Alguns pontos podem ser ressaltados no que diz respeito à elevada similaridade florística entre ambos os estudos: no caso de Leguminosae, todas as quatro espécies encontradas nesse estudo também ocorreram em Jurubatiba. Ainda, as duas espécies de Rubiaceae encontradas no PARNA não amostradas nesse estudo foram incluídas na listagem florística da APA de Massambaba (Araujo et al. 2009) como ocorrentes na formação analisada (Mitracarpus frigidus (Willd.) K. Schum.) e em outra formação arbustiva aberta próxima (Chioccoca alba (L.) Hitchc.).

Por outro lado, apesar de algumas ocorrerem em ambas as comunidades, elas não apresentam a mesma importância na estrutura dessas formações vegetais. Por exemplo, Neoreogelia cruenta, espécie que ocorreu em 15,5\% das parcelas de Jurubatiba, apresentando $6,82 \%$ da cobertura vegetal, no presente estudo ocorreu em apenas uma parcela (frequência relativa $=0,12 \%$ ) e representou $0,41 \%$ da cobertura vegetal encontrada para Massambaba.

Em relação à diversidade, observa-se que o estrato herbáceo de Massambaba apresentou maior valor do índice de Shannon baseado em cobertura $\left(\mathrm{H}^{\prime} \mathrm{C}\right)$ quando comparado com o de Jurubatiba (Tab. 1), sendo tal diferença estatisticamente significativa $(\mathrm{p}<0,001)$. Uma vez que a diversidade de Shannon baseia-se na riqueza de espécies e na abundância, o alto valor deste índice no presente trabalho confirma a elevada riqueza de espécies vegetais encontrada no Centro de Diversidade Vegetal de Cabo Frio, quando comparado com as demais áreas do litoral fluminense (Araujo 1997). Os estudos realizados nessa região têm ressaltado o elevado número de espécies de diversos grupos, como algas (Brasileiro et al. 2009) e angiospermas (Sá 2006), assim como o alto endemismo, abrigando $65 \%$ das espécies vegetais endêmicas das restingas do estado do Rio de Janeiro consideradas por Araujo (2000).

Analisando o índice de diversidade de Shannon, Pereira et al. (2004) apontaram que o valor de cobertura é a melhor opção para substituir o número de indivíduos no cálculo de H' por melhor representar a estrutura oligárquica, assim como apontam que a utilização da frequência pode subestimar a abundância das espécies mais comuns, aumentando a equabilidade da comunidade. De fato, neste trabalho, o valor do índice de diversidade baseado na frequência $\left(\mathrm{H}^{\prime}{ }_{\mathrm{F}}\right)$ foi superior do que o baseado em cobertura $\left(\mathrm{H}^{\prime}{ }_{\mathrm{F}}=2,90 \times \mathrm{H}_{\mathrm{C}}{ }=2,32\right)$, assim como em Jurubatiba, mas essa diferença não foi tão abrupta como os valores encontrados por Pereira et al. (2004) $\left(\mathrm{H}_{\mathrm{F}}{ }_{\mathrm{F}}=3,01 \times \mathrm{H}^{\prime}{ }_{\mathrm{C}}=1,89\right)$. Essa menor diferença sugere que a proporção entre as coberturas das espécies é maior em Massambaba, o que se confirma ao comparar ambos valores de equabilidade $\left(\mathrm{J}^{\text {C Massambaba }}=0,67 \times \mathrm{J}_{\text {C Jurubatiba }}=0,52\right)$.

A elevada heterogeneidade em Jurubatiba é explicada pela grande dominância de uma única espécie: Allagoptera arenaria, que apresentou 50,95\% da Cobertura Relativa. Já em Massambaba, são necessárias duas espécies para somar valor próximo de CR (47,85\%): Panicum trinii e $A$. arenaria, o que explica a maior a equabilidade encontrada nessa comunidade.

As comparações aqui realizadas demonstram a alta similaridade florística entre a formação arbustiva aberta não inundável (fácies alta) da APA de Massambaba e a formação aberta de Clusia, no Parque Nacional da Restinga de Jurubatiba, além da 
semelhança estrutural entre as comunidades, uma vez que em ambas, a vegetação está organizada em moitas de espécies arbustivas rodeadas por areia nua e vegetação esparsa. Entretanto, a ausência de outros trabalhos com metodologia padronizada e mesma definição de estrato herbáceo, limita inferir sobre os fatores que explicam o padrão de ocorrência das espécies levantadas, assim como a possível influência de pressões antrópicas sobre tais comunidades vegetais.

\section{Agradecimentos}

À Dorothy S.D. Araujo e à Viviane S. Fonseca-Kruel, pela contribuição na formulação do projeto e desenvolvimento do trabalho. À Miriam C.A. Pereira pelas sugestões e críticas à monografia da primeira autora, que foram incorporadas neste manuscrito. Ao Robson D. Ribeiro (in memorian), à Adriana C.S. Cavalcanti e ao Jorge Caruso pela valiosa ajuda no campo. À FAPERJ, pela bolsa de iniciação científica concedida à primeira autora. Aos especialistas que auxiliaram na identificação do material botânico: Marcus Nadruz, Rafaella C. Forzza, Débora Medeiros, Robson D. Ribeiro, Eduardo M. Saddi, Claudine M. Mynssen e Fátima R.G. Salimena.

\section{Referências}

Almeida, A.L. \& Araujo, D.S.D. 1997. Comunidades vegetais do cordão arenoso externo da Reserva Ecológica Estadual de Jacarepiá, Saquarema, RJ. In: Absalão, R.S. \& Esteves, A.M. (eds.). Ecologia de praias arenosas do litoral brasileiro. Oecologia Brasiliensis Séries, Vol. 3. PPGE-UFRJ, Rio de Janeiro. Pp. 47-63.

Alves, R.J.V.; Cardin, L. \& Kropf, M.S. 2007. Angiosperm disjunction "campos rupestres - restingas": a reevaluation. Acta Botânica Brasílica 21: 675-685.

Angiosperm Phylogeny Group II (APG II). 2003. An up date of the Angiosperm Phylogeny Group classification for the orders and families of flowering plants: APGII. Botanical Journal of Linnaean Society 141: 399-436.

Araujo, D.S.D. 1997. Cabo Frio Region. In: Davis, S.D.; Heywood, V.H.; Herrera-MacBryde, O.; VillaLobos, J. \& Hamilton, A.C. (eds.). Centres of plant diversity: a guide and strategy for their conservation. Vol. 3: The Americas. IUCN Publications Unit, Cambridge. Pp. 373-375.

Araujo, D.S.D. 2000. Análise florística e fitogeográfica das restingas do estado do Rio de Janeiro. Tese de Doutorado. Universidade Federal do Rio de Janeiro, Rio de Janeiro. 176p.
Araujo, D.S.D. \& Maciel, N.C. 1998. Restingas fluminenses: biodiversidade e preservação. Boletim FBCN 25: 27-51.

Araujo, D.S.D.; Pereira, M.C.A. \& Pimentel, M.C.P. 2004. Flora e estrutura de comunidades no Parque Nacional da Restinga de Jurubatiba - Síntese dos conhecimentos com enfoque especial para formação aberta de Clusia. In: Rocha, C.F.D.; Esteves, F.A. \& Scarano, F.R. (orgs.). Pesquisas de longa duração na restinga de Jurubatiba: ecologia, história natural e conservação. RiMa, São Carlos. Pp. 59-76.

Araujo, D.S.D; Sá, C.F.C.; Fontella-Pereira, J.; Garcia, D.S.; Ferreira, M.V.; Paixão, R.J.; Schneider, S.M. \& Fonseca-Kruel, V.S. 2009. Área de Proteção Ambiental de Massamababa, Rio de Janeiro: caracterização fitofisionômica e florística. Rodriguésia 60: 67-96.

Assumpção, J. \& Nascimento, M.T. 2000. Estrutura e composição florística de quatro formações vegetais de restinga no complexo lagunar Grussaí/Iquipari, São João da Barra, RJ, Brasil. Acta Botanica Brasilica 14: 301-315.

Barbiere, E.B. 1984. Cabo Frio e Iguaba Grande, dois microclimas distintos a um curto intervalo espacial. In: Lacerda, L.D.; Araújo, D.D.D.; Cerqueira, R. \& Turcq, B. (eds.). Restingas: origem, estrutura, processos. CEUFF, Niterói. Pp. 3-13.

Bohrer, C.B.A.; Dantas, H.G.R.; Cronemberger, F.M.; Vicens, R.S. \& Andrade, S.F. 2009. Mapeamento da vegetação e uso do solo mo Centro de Diversidade Vegetal de Cabo Frio, Rio de Janeiro, Brasil. Rodriguésia 60: 1-24.

Brasileiro, P.S.; Yoneshigue-Valentin, Y.; Bahia, R.G.; Reis, R.P. \& Amado Filho, G.M. 2009. Algas marinhas bentônicas da região de Cabo Frio e arredores: síntese do conhecimento. Rodriguésia 60: 39-66.

Brower, J.E.; Zar, J.H. \& VonEnde, C.N. 1998. Field \& laboratory methods for general ecology. 4 ed. McGraw-Hill, Boston. Pp 90-96.

Campello, E.F.C. 1998. Sucessão vegetal na recuperação de áreas degradas. In: Dias, L.E. \& Mello, J.W.V. (eds.). Recuperação de áreas degradas. Ed. Folha de Viçosa, Viçosa. Pp. 183-196.

Cerqueira, R. 2000. Biogeografia das restingas. In: Esteves, F.A. \& Lacerda, L.D. (eds.). Ecologia de restingas e lagoas costeiras. NUPEM/UFRJ, Macaé. Pp. 65-75.

Cirne, P.; Zaluar, H.L.T. \& Scarano, F.R. 2003. Plant diversity, interespecific associations and postfire resprouting on a sandy spit in a Brazilian coastal plain. Ecotropica 9: 33-38.

Citadini-Zanette, V. \& Baptista, L.R.M. 1989. Vegetação herbácea terrícola de uma comunidade florestal em Limoeiro, município de Torres, Rio Grande do Sul, Brasil. Boletim do Instituto de Biociências da Universidade Federal do Rio Grande do Sul 45: 1-87. 
Cordeiro, S.Z. 2005. Composição e distribuição florística da vegetação herbácea em três áreas com fisionomias distintas na Praia do Peró, Cabo Frio, RJ, Brasil. Acta Botanica Brasilica 19: 679-693.

Davis, S.D.; Heywood, V.H.; Herrera-MacBryde, O.; Villa-Lobos, J. \& Hamilton, A.C. (eds). 1997. Centers of plant diversity: a guide and strategy for their conservation. Volume 3: The Americas. IUCN Publications Unit, Cambridge. 552p.

Felfili, J.M.; Silva Junior, M.C.F.; Rezende, A.V.; Haridasan, M.; Filgueiras, T.S.; Mendonça, R.C.; Walter, B.M.T. \& Nogueira, P.E. 2001. O projeto Biogeografia do Bioma Cerrado: hipóteses e padronização da metodologia. In: Garay, I. \& Dias, B. (orgs.). Conservação da biodiversidade em ecossistemas tropicais. Vozes, Petrópolis. Pp. 157-163.

Fonseca-Kruel, V.S.; Araújo, D.S.D.; Sá, C.F.C. \& Peixoto, A.L. 2009. Quantitative ethnobotany of a restinga fragment in Rio de Janeiro, Brasil. Rodriguésia 60: 187-202.

Fraga, C.N.; Kollmann, L.J.C. \& Menezes, L.F.T. 2005. Orchidaceae da Restinga de Marambaia, Rio de Janeiro, RJ. In: Menezes, L.F.T., Peixoto, A.L. \& Araujo, D.S.D. (eds.). Histórianatural da Marambaia. EDUR, Seropédica. Pp. 121-132.

Freitas, M.F. 1992. Cactaceae da Área de Proteção Ambiental da Massambaba, Rio de Janeiro, Brasil. Rodriguésia 42/44: 67-91.

Freitas, A.F.N.; Cogliatti-Carvalho, L.; Vansluys, M. \& Rocha, C.F.D. 2000. Distribuição espacial de bromélias na restinga de Jurubatiba, Macaé, RJ. Acta Botanica Brasilica 14: 127-242.

Hay, J.D. \& Lacerda, L.D. 1984. Ciclagem de nutrientes do ecossistema de restinga. Pp. 459-475. In: Lacerda, L.D.; Araújo, D.S.D.; Cerqueira, R. \& Turcq, B. (orgs.). Restingas: origem, estrutura, processos. CEUFF, Niterói.

Henriques, R.P.B.; Araujo, D.S.D. \& Hay, J.D. 1986. Descrição e classificação dos tipos de vegetação da restinga de Carapebus, Rio de Janeiro. Revista Brasileira de Botânica 9: 173-189.

Kneip, L.M. \& Pallestrini, L. 1984. Restingas do estado do Rio de Janeiro (Niterói a Cabo Frio): 8 mil anos de ocupação humana. In: Lacerda, L.D.; Araújo, D.S.D.; Cerqueira, R. \& Turcq, B. (orgs.). Restingas: origem, estrutura, processos. CEUFF, Niterói. Pp. 139-148.

Lacerda, L.D.; Araujo, D.S.D. \& Maciel, N.C. 1993. Dry coastal ecosystems of the tropical brazilian coast. In: Maarel, E.V.D. (ed.). Dry coastal ecosystems: Africa, America, Asia and Oceania. Elsevier, Amsterdan. Pp. 477-493.

Lewis, G.P.; Schrire, B.; Mackinder, B. \& Lock, M. (eds.). 2005. Legumes of the world. Royal Botanic Gardens, Kew. 577p.
Magurran, A.E. 1988. Ecological diversity and its measurement. Princeton University Press, New Jersey. 179p.

Menezes, L. F. T. \& Araujo, D.S.D. 1999. Estrutura de duas formações vegetais do cordão externo da Restinga de Marambaia, RJ. Acta Botânica Brasilica 13: 223-235.

Menezes, L.T.F. \& Araujo, D.S.D. 2004. Regeneração e riqueza da formação arbustiva de Palmae em uma cronosequência pós-fogo na Restinga da Marambaia, Rio de Janeiro, RJ, Brasil. Acta Botanica Brasilica 18: 771-780.

Montezuma, R.C.M. \& Araújo, D.S.D. 2007 Estrutura da vegetação de uma restinga arbustiva inundável no Parque Nacional da Restinga de Jurubatiba, Rio de Janeiro. Pesquisas Botânica 58: 157-176

Muehe, D. 1994. Lagoa de Araruama: geologia e sedimentação. Cadernos de Geociências do IBGE 10: 53-62.

Mueller-Dombois, D. \& H. Ellenberg. 1974. Aims and methods of vegetation ecology. John Wiley \& Sons, New York. 118p.

Pereira, M.C.A.; Araujo, D.S.D. \& Pereira, O.J. 2001 Estrutura de uma comunidade arbustiva da restinga de Barra de Maricá, RJ. Revista Brasileira de Botânica 24: 273-281.

Pereira, M.C.A.; Cordeiro, S.Z. \& Araujo, D.S.D. 2004. Estrutura do estrato herbáceo na formação aberta de Clusia do Parque Nacional da Restinga de Jurubatiba, RJ, Brasil. Acta Botanica Brasilica 18: 677-687.

Ribeiro, R.D. \& Lima, H.C. 2009. Riqueza e distribuição geográfica de espécies arbóreas da família Leguminosae e implicações para conservação no Centro de diversidade vegetal de Cabo Frio, Rio de Janeiro, Brasil. Rodriguésia 60: 111-128.

Rocha, C.F.D.; Bergallo, H.G.; Alves, M.A.S. \& Sluys, M.V. 2003. A biodiversidade nos grandes remanescentes florestais do estado do Rio de Janeiro e nas restingas da Mata Atlântica. RiMa, São Carlos. Pp. 85-88.

Rocha, C.F.D.; Bergallo, H.G.; Alves, M.A.S. \& Jamel, C.E. 2007. The remnants of restinga habitats in the brazilian Atlantic Forest of Rio de Janeiro state, Brazil: habitat loss and risk of disappearance. Brazilian Journal of Biology 67: 263-273.

Rocha, C.F.D.; Castro, T.C.C.; Teixeira, A.M.G.; Cruz, C.B.M.; Figueiredo, C.A.A.; Bohrer, C.B.A.; Volcker, C.M.; Sá, C.F.C.; Chaves, L.C.T.; Maciel, N.C. \& Villaça, R.C. 2009. Região turística dos lagos fluminenses. In: Bergallo, H.G.; Fidalgo, E.C.C.; Rocha, C.F.D.; Uzêda, M.C.; Costa, M.B.; Alves, M.A.S.; Sluys, M.V.; Santos, M.A.; Costa, T.C.C. \& Cozzolino, A.C.R. (orgs.). Estratégias e ações para a conservação da biodiversidade no estado do Rio de Janeiro. Instituto Biomas, Rio de Janeiro. Pp. 313-323. 
Rocha-Pessôa, T.C.; Nunes-Freitas, A.F.; Cogliatti, L. \& Rocha, C.F.D. 2008. Species composition of Bromeliaceae and their distribution at the Massambaba restinga in Arraial do Cabo, Rio de Janeiro, Brazil. Brazilian Journal of Biology 68: 251-257.

Sarahyba, L.S.P. 1993. Gramineae (Poaceae) da Área de Proteção Ambiental de Massambaba, Rio de Janeiro, Brasil. Dissertação de Mestrado. Museu Nacional, Rio de Janeiro. 145p.

Sá, C.F.C. 1996. Regeneração em uma área de floresta de restinga na Reserva Ecológica Estadual de Jacarepiá, Saquarema, estado do Rio de Janeiro: I - Estrato herbáceo. Arquivos do Jardim Botânico do Rio de Janeiro 34: 177-192.

Sá, C.F.C. 2002. Regeneração de um trecho de floresta de restinga na Reserva Ecológica Estadual de Jacarepiá, Saquarema, estado do Rio de Janeiro: II - Estrato arbustivo. Rodriguésia 53: 5-23.

Sá, C.F.C. 2006. Estrutura, diversidade e conservação de angiospermas no Centro de Diversidade de Cabo Frio, estado do Rio de Janeiro. Tese de Doutorado. Universidade Federal do Rio de Janeiro, Rio de Janeiro. 250p.

Sá, C.F.C. \& Araújo, D.S.D. 2009. Estrutura e florística de uma floresta de restinga em Ipitangas, Saquarema, Rio de Janeiro, Brasil. Rodriguésia 60: 147-170.

Scarano, F.R. 2002. Structure, function and floristic relationships of plant communities in stressful habitats marginal to the Brazilian Atlantic Rainforest. Annals of Botany 90: 517-524.

Scarano, F.R.; Cirne, P.; Nascimento, M.T.; Sampaio, M.C.; Vilela, D.M.; Wendt, T. \& Zaluar, L.T. 2004. Ecologia vegetal: integrando ecossistema, comunidades, populações e organismos. In: Rocha, C.F.D.; Esteves, F.A. \& Scarano, F.R. (orgs.). Pesquisas de longa duração na restinga de Jurubatiba: ecologia, história natural e conservação. RiMa, São Carlos. Pp.77-97.

SEMADS/RJ - Secretaria de Meio Ambiente e Desenvolvimento Sustentável do Rio de Janeiro. 2001. Atlas da Unidade de Conservação da Natureza do Estado do Rio de Janeiro. Metalivros, São Paulo. Pp. 42-43.

Silva, J.G. \& Somner, G.V. 1984. A vegetação de restinga na Barra de Maricá, RJ. In: Lacerda, L.D.; Araújo, D.S.D.; Cerqueira, R. \& Turcq, B. (orgs.). Restingas: origem, estrutura, processos. CEUFF, Niterói. Pp. 217-225.

Smith, A.R.; Pryer, K.M.; Schuettpelz, E.; Korall, P.; Schneider, H. \& Wolf, P.G. 2006. A classification for extant ferns. Taxon 55: 705-731.

Soares-Gomes, A. \& Fernandes, F.C. 2005. Spatial distribution of bivalve mollusc assemblages in the upwelling ecosystem of the continental shelf of Cabo Frio, Rio de Janeiro, Brazil. Revista Brasileira de Zoologia 22: 73-80.

Yadav, A.S. \& Gupta, S.K. 2007. Effect of microenvironment and human disturbance on the diversity of herbaceous species in Sariska Tiger Project. Tropical Ecology 48: 125-128.

Zaluar, H.L.T. \& Scarano, F.R. 2000. Facilitação em restingas de moitas: um século de buscas por espécies focais. In: Esteves, F.A. \& Lacerda, L.D. (eds.). Ecologia de restingas e lagoas costeiras. NUPEM/ UFRJ, Macaé. Pp. 3-23.

Zar, J.H. 1999. Biostatistical analysis. 4 ed. PrenticeHall, New Jersey. Pp. 156-158. 